\title{
APORTACIONES AL CONOCIMIENTO DEL GENERO EQUISETUM L. I. DESCRIPCION DE LOS MICROCARACTERES DE E. PALUSTRE L.
}

\author{
J. IRANZO, M. VILAR \& A. E. SALVO
}

\begin{abstract}
RESUAEN: Se realiza una descripción detallada de los caracteres anatomo-histológicos de Equisetum palustre $L$.
\end{abstract}

RESUME: On réalise une description détaillée des caractères anatomo-histologiques de Equisetum palustre L.

\section{INTRODUCCION}

Es evidente que dentro de la taxonomía actual existe una clara tendencia al estudio de los microcaracteres, no sólo por cuanto estos nos van a permitir una mejor estructuración de los táxones, sino que además por su fijeza suelen ser excelentes caracteres diferenciadores. Sin embargo, los microcaracteres suelen gozar de cierta mala fama sobre todo cuando son utilizados para el diagnóstico, ya que suelen ser "incómodos" de observar.

Dentro de la pteridología es cada vez más frecuente la utilización de caracteres tales como morfología y diámetros esporales, morfología y diámetros estomáticos, o incluso la determinación del número cromosomático, para lograr un pronunciamiento preciso sobre el binomen a adjudicar a un especimen. Especialmente significativo es el caso del género Equisetum. Milde (1867) ya sentencia en su lapidaria frase "sine examine microscopia nulla scientia equisetorum", que tan sólo el estudio de los microcaracteres puede garantizar una buena determinación. Muchos han sido los intentos posteriores en los que se han pretendido claves de determinación en las que por ofertar una mayor comodidad de diagnosis, se ha abundado en caracteres de fácil apreciación cuyo grado de fiabilidad es considerablemente pobre. Por desgracia los autores de estas tentativas no insistieron en la línea marcada por Milde, que aunque afinó bastante en sus investigaciones, las limitaciones técnicas de la época no le permitieron la precisión deseada. Es pues nuestro objetivo en esta serie de artículos redescribir los caracteres anatomo-morfológicas de cada uno de los táxones de este grupo vegetal. 


\section{METODO}

Aunque somos conscientes de que la norma general es que la determinación de los especímenes recolectados se va a realizar una vez desecados estos, hemos creido conveniente realizar nuestros ensayos sobre material lo más intacto posible, ya que es la única forma posible de llegar a una aproximación lo más real, y permite realizar las extrapolaciones pertinentes a material seco. Para ello, cuando el material se localizaba cercanamente, procedíamos a transportarlo al laboratorio en nevera a $5-10^{\circ} \mathrm{C}$. En el caso de que la recolección se efectuara lejanamente, fijábamos en material. directamente en el campo. El fijador elegido, por su bondad y sobre todo por las escasas alteraciones que producía, ha sido la mezcla a partes iguales de etanol-glicerina-agua. Para la obtención de cortes utilizamos el microtomo de congelación, variando el grosor de estos entre 20-40 $\mu \mathrm{m}$. Finalmente, como tinción hemos preferido la de verde-rápido-safranina, por cuanto era la que aportaba mayor contraste y limpieza.

\section{EQUISETUM PALUSTRE L.}

Para iniciar nuestra serie hemos elegido este taxon por ser, dentro del género, el que con mayor frecuencia aparece erróneamente determinado en los herbarios, confundiéndose normalmente con Equisetum arvense L., al cual recuerda por su hábito, a lo que colabora el hecho de que ocasionalmente $\mathrm{E}$. arvense L. presente conos estrobilares en los tallos estériles, fenómeno no tan raro como muchos autores han querido ver. Buenas descripciones de los macrocaracteres se hallan en Hauke (1978) y Page (1982).

\section{DESCRIPCION ANATOMO-HISTOLOGICA}

Rizoma. Antetodo es necesario hacer una serie de puntualizaciones terminológicas que creemos son de gran interés. Aplicamos aqui el término de rizoma exclusivamente a aquella parte del tallo subterráneo, de varios años de edad, caracterizada fundamentalmente por la presencia de raices en los nudos (en vez de micrófila, como es el caso de los vástagos aéreos) y que por tratase de órganos de reserva, y por tanto engrosados, la superficie de los entrenudos no va a presentar una diferenciación en carenas y valléculas, es decir presentará un contorno netamente circular. Esta precisión la realizamos con el fin de diferenciar claramente al rizoma de aquellos otros vástagos subterráneos, anuales, cuyo fin es la emersión de los nuevos vástagos aéreos.

Por otra parte, la ausencia de diferenciación en valléculas y carenas en la superficie de los rizomas de Equisetum, nos lleva a replantearnos la denominación de dos estructuras histológicas basadas precisamente en su situación respecto a la topografía externa. Concretamente nos referimos a los canales valleculares y a los canales carinales. Los primeros, con un origen lisogénico de las células corticales, preferimos denominarlos "canales corticales", mientras que los segundos, desarrollados lisoesquizogénicamente, "canales vasculares".

En el caso concreto de Equisetum palustre L. el rizoma presenta un alto valor taxonómico (Lám. 1-A). Este presenta externamente 
un color castaño-oscuro a negruzco, debido a que la epidermis se encuentra totalmente suberificada. Con bastante frecuencia esta suberificación suele alcanzar a las capas de células mas externas del parénquima cortical. Este parénquima cortical se encuentra considerablemente reducido a 4-6 estratos de células, las cuales se carac terizan por presentar numerosos amiloplastos. Los canales corticales son de un gran tamaño y ocupan el $50 \%$ o más de la superficie transversal del rizoma, lo cual sirve para diferenciarlo de E. fluviatile L., ya que este no presenta dichos canales. Estos, están separados entre si por tractos celulares del parénquima interlagunar formados por 3-4 filas de células, que une el parénquima cortical con el cilindro central. Separando el cilindro central y el córtex aparece una banda de Caspary bien desarrollada (Lám. 1-B) que rodea exteriormente a todos los haces conductores. En contraposición con la idea de Bierhorst (1958) de que no existe una diferenciación entre proto y metaxilema en Equisetum, hemos de afirmar que a nuestro parecer, y en concreto en el caso de los rizomas, los más normal es que se desarrolle exclusivamente protoxilema, debidoal escaso crecimiento que sufren los entrenudos, aunque raramente hemos podido observar desarrollo metaxilemático. Como puede verse (Lám. 1-A) Equisetum palustre carece totalmente de canal medular en el rizoma, siendo este el mejor carácter distintivo por cuanto lo diferencia de todas las demás especies del género.

Vástagos aéreos. La epidermis de los vástagos aéreos de E. palustre está constituida por células que presentan en su superficie externa una gran cantidad de pílulas de sílice (Lám. 1-C), sobre todo a nivel de las valléculas. También aquí suelen aparecer otros orgánulos, mamelas silíceas, las cuales se disponen ordenada y transversalmente y que en comparación son más inconspicuas que en otros equisetos.

En cuanto a la disposición de los estomas, esta resulta bastante desigual a lo largo de los tallos aéreos, dependiendo fundamentalmente de su situación altitudinal. Asi, mientras que en los primeros entrenudos los estomas son escasos, disponiéndose en dos dobles filas en las valléculas (de forma similar a E. arvense L.), a medida que ascendemos a los entrenudos superiores aumenta el número y llegan a disponerse incluso hasta en 12-14 filas, ocupando entonces toda la superficie vallecular. Estas observaciones quedan demostradas en los resultados obtenidos tras aplicar el indice estomático $\left(\mathrm{Ie}=\mathrm{N}^{\circ}\right.$ estomas/ $\mathrm{N}^{\circ}$ células epidérmicas + estomas) a las regiones valleculares. De esta forma podemos observar como el primer segmento del tallo presenta una gran variabilidad longitudinal, de tal manera que en las regiones basales el valor de le alcanzaba tan sólo 0,03, mientras que en las distales, en contacto con la vaina superior, llegaba hasta 0,09. El segundo artejo presenta un Ie de 0,23, sin apreciarse grandes variaciones longitudinales. El tercer segmento posee un índice un poco mayor, del orden de 0,27, aumentando en el cuarto a 0,28 y en el quinto a 0,33 , valor que se aproxima al de los segmentos superiores, así en los segmentos sexto, séptimo y octavo el valor del índice es ya de 0,34 .

En lo referente a la distribución del colénquima disentimos con la mayoría de los autores en el sentido de que si bien este aparece formando columnas carinales, en los ejemplares robustós suelen aparecer cordones colenquimatosos valleculares que, interrumpen el clorénquima e interfieren en la disposición y número de los estomas, tanto en los entrenudos basales como en los próximos a los conos estrobilares ( $L a ́ m$. 1-D,E y F). La aparición de este colénquima vallecular parece estar directamente relacionada con hábitats secos. 
El córtex se encuentra formado por parénquima cortical, constituido por clorénquima y parénquima interlagunar. Este último se caracteriza por carecer de cloroplastos, o contener muy pocos, y por el contrario poseer numerosos amiloplastos, carácter este bastante bueno para diferenciarlo de Equisetum arvense L.

Las lagunas corticales (Lám. 2-A) presentan gran variabilidad en cuanto a su tamaño, siendo bastante pequeñas en los primeros entrenudos y aumentando progresivamente hacia el ápice donde pueden ocupar hasta el $40 \%$ de la sección del entrenudo.

En cilindro central (Lám. 2-B y C) presenta periféricamente una endodermis en la que pueden distinguirse una banda de Caspary y más hacia el interior un periciclo de células más o menos prismáticas bien desarrolladas. Los haces conductores están más desarrollados que en rizoma y si presenta aquí un metaxilema bien constituido. Por el contrario, el protoxilema prácticamente desaparece y deja de ser funcional al quedar destruido por elongación de los entrenudos. Unicamente se pueden encontrar restos de este protoxilema en las lagunas vasculares. La laguna medular, que se forma por una destrucción desigual del parénquima medular es relativamente pequeña y de morfología variable.

Vainas de micrófilas. Las micrófilas que en número de 5-12 se disponen formando las vainas de los nudos, presentan dos filas de estomas que se disponen a ambos lados del nervio medio. Esta disposición se debe al hecho de que centralmente en cada micrófila aparece una banda de colénquima más o menos desarrollada (Lám. 2-D). Sin embargo, a nivel de las vainas rameales este colénquima está ausente.

En la región de fusión de la micrófilas, y más concretamente en la zona media de dicha fusión, aparecen unas células especiales denominadas "anquirocitos" (Lám. 2-E). La presencia de estas células nos sirve para diferenciar a E. palustre de E. sylvaticum y E. fluviatile, por cuanto estos no las presentan.

Ramificaciones laterales. Las ramas laterales de E. palustre presentan una organización semejante a la del tallo. En cualquier caso deben destacarse los siguientes caracteres distintivos. En las ramas primarias los cordones colenquimatosos, que son muy reducidos, se restringen a las carenas, mientras que las ramas secundarias no presentan ningún tipo de colénquima. El canal medular de las ramas es en relación al tallo bastante grande. En las ramas prácticamente el protoxilema ha desaparecido, mientras que el metaxilema se encuentra muy desarrollado. Finalmente, es necesario resaltar la presencia de canales corticales en las ramas de Equisetum palustre, lo cual es un buen caracter para separarlo de E. sylvaticum, E. fluviatile y sobre todo de E. arvense (Lám. 2-F y G).

\section{MATERIAL ESTUDIADO}

ASTURIAS: Carretera de Oviedo-Santander, Km 161, 29.VI.1984, Salvo et al. (MGC 14322). LEON: Subida al Puerto de Somiedo, 28.VI.1984, Salvo et al. (MGC 14299). VALENCIA: Ribarroja del Turia, 1.V.1984, Vilar (MGC 13695). 

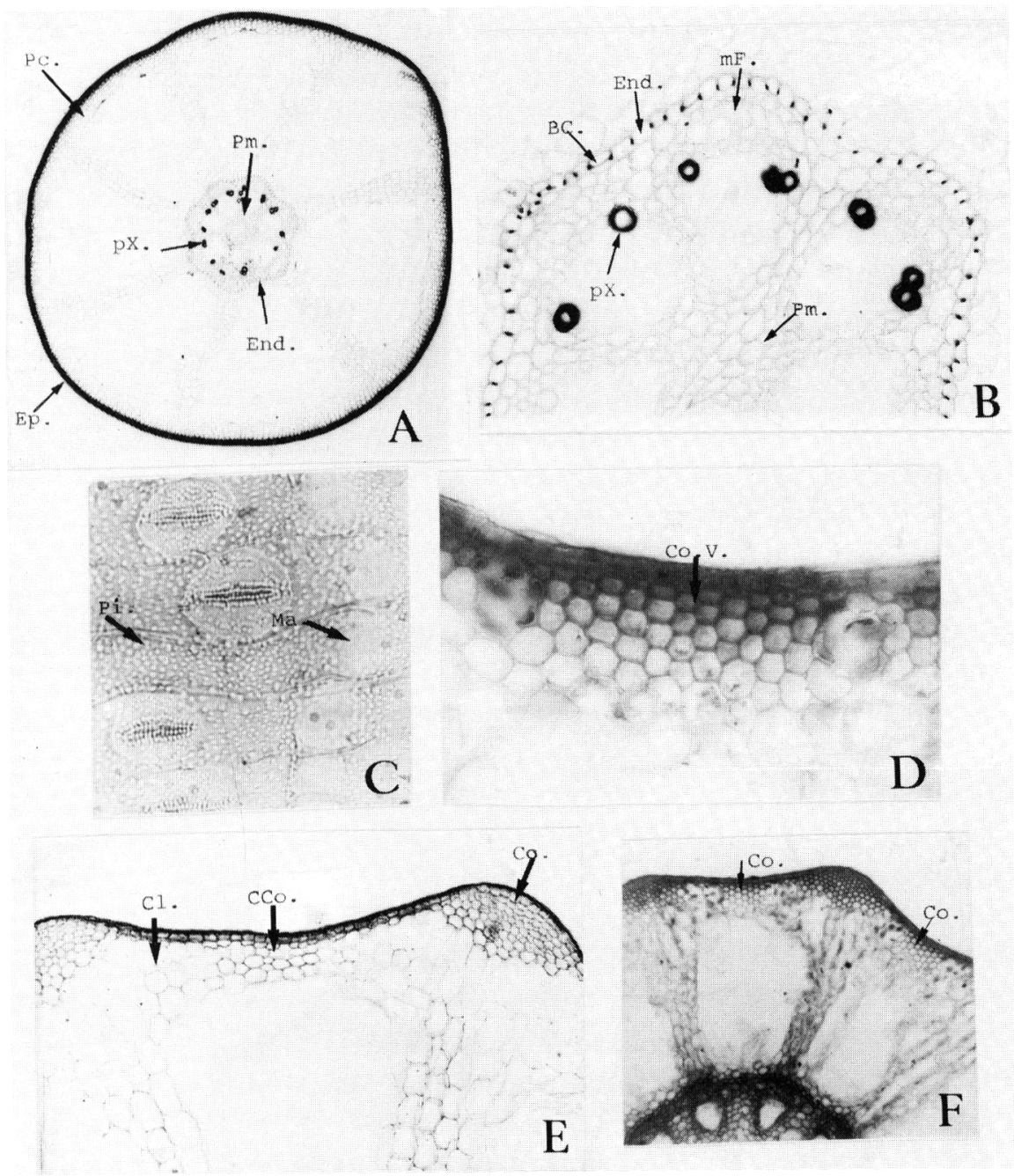

Lámina 1.- A: Sección del rizoma. B: Cilindro central del rizoma. C: Epidermis. D: Detalle de las valléculas del primer entrenudo del vástago aéreo. E: Detalle de las valléculas del tercer entrenudo del vástago aéreo. F: Apreciación de la distribución del colénquima en los vástagos aéreos.

Clave de abreviaturas.- An: Anquirocitos. Bc: Banda de Caspary. Cco: Células colenquimáticas. Cl: Clorénquima. Co: Colénquima. Cov: Colénquima vallecular. End: Endodermis. Ep : Epidermis. Lc: Laguna cortical. Lm: Laguna medular. Ma: Mamelas siliceas. mF: Metafloema. mX: metaxilema. Pc: Parénquima cortical. Per: Periciclo. Pi: Pilulas silíceas. Pm: Parénquima medular. Px: Protoxilema. 

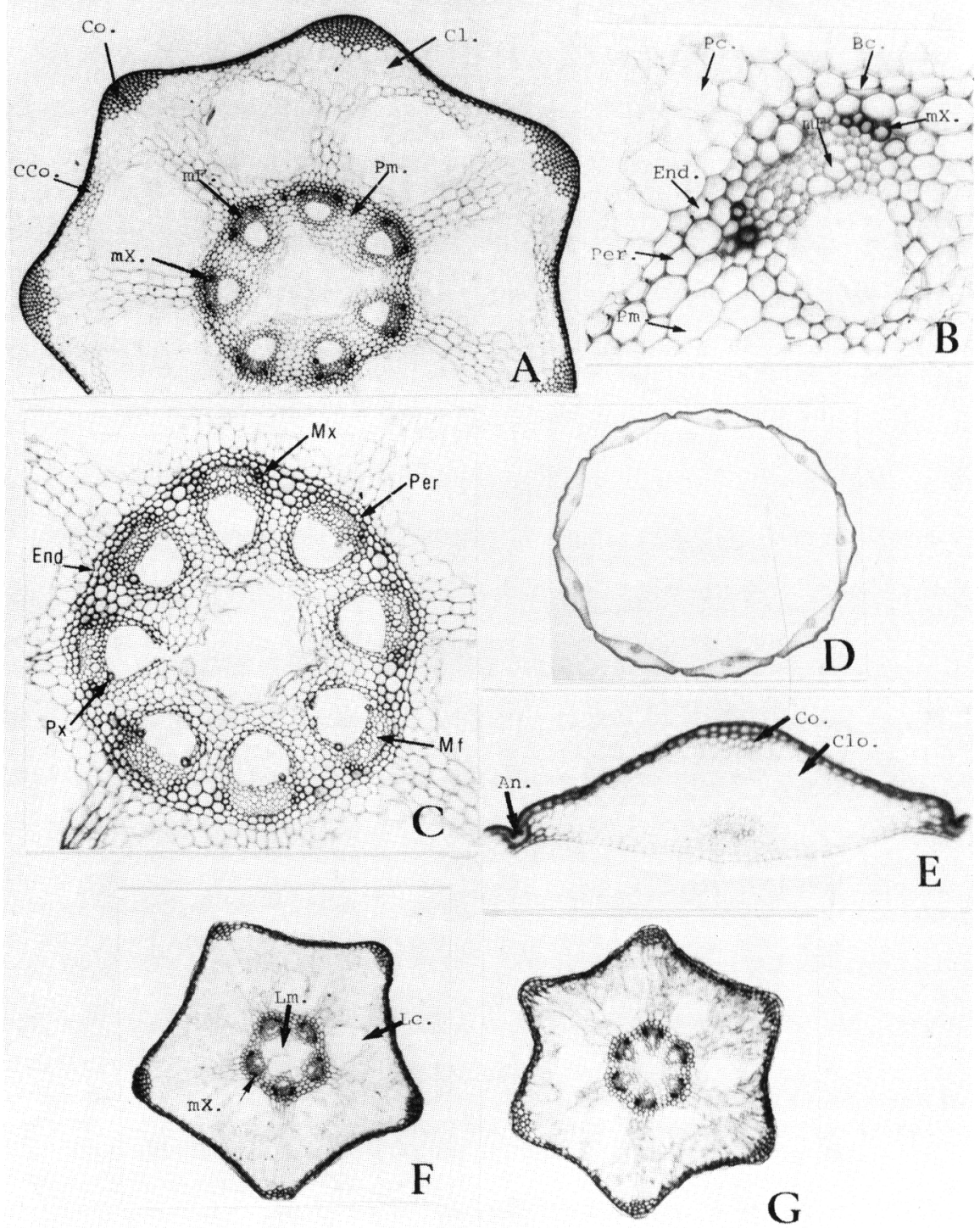

Lámina 2.- A: Sección del vástago aéreo. B: Haz del vástago aéreo. C: Cilindrocentral del vástago aéreo. D: Sección de la vaina del tallo principal. E: Sección de una micrófila. $F$ y G: Secciones transversales de entrenudo rameales. 


\section{BIBL IOGRAFIA}

BIERHORST, D.W. -1958- Vessels in Equisetum. Amer. Jour. Bot., 45: 534537.

HAUKE, R.L. -1978- A taxonomic monograph of Equisetum subgenus Equisetum. Nova Hedwigia, 30: 385-451.

MILDE, J. -1867- Monografia Equisetorum. Nova Acta Peop.-Carol., 32(2).

PAGE, C. N. -1982- The ferns of Britain and Ireland. Cambridge University Press.

Dirección de los autores: J. IRANZO y M. VILAR, Departamento de Botánica, Facultad de Farmacia, Univ. de Valencia. A.E. SALVO, Departamento de Botánica, Facultad de Ciencias, Univ. de Málaga. 